\title{
Intestinal FDG-PET/CT imaging of an Eritrean with schistosomiasis seen in Denmark
}

\author{
Ata Daghigh* ${ }^{*}$, Julie Marie Grüner and Peter Mørup
}

\author{
*Correspondence: ata.daghigh@ \\ gmail.com \\ Department of Clinical Physiology \\ and Nuclear Medicine, Zealand \\ University Hospital, Lykkebækvej 1, \\ 4600 Køge, Denmark
}

\begin{abstract}
Background: Schistosomiasis is one of the most common parasitic diseases in subtropical and tropical areas and still is considered of public health significance. This disease affects about 200 million people around the world. Intestinal schistosomiasis is mainly diagnosed by parasitological, serological, and molecular methods.

Case presentation: A 36-year-old Eritrean man who had lived in Denmark for the past 3 years presented to the hospital with 4 months' history of abdominal pain, back pain, and weight loss of $12 \mathrm{~kg}$. He underwent 18F-FDG-PET/CT scanning. The scan findings were consistent with schistosomiasis, which were confirmed by serological and pathological tests.

Conclusion: PET/CT is a common modality neither to detect schistosomes nor to diagnose schistosomiasis. A presumptive diagnosis can be made based on coincidence of high FDG uptake in visceral lymph nodes below the diaphragm and in relation to abdominal viscera, travel history suggestive of schistosome infection, and exclusion of other causes of abdominal pain.

Keywords: Intestinal parasite, Schistosoma mansoni, FDG-PET/CT
\end{abstract}

\section{Background}

Five schistosome species can cause infection in humans (Table 1). The incubation period for patients with acute schistosomiasis is usually 14-84 days; however, many people are asymptomatic and have subclinical disease during both acute and chronic stages of infection. Persons with acute infection (also known as Katayama syndrome) may present with rash, fever, headache, myalgia, and respiratory symptoms. Often eosinophilia is present with hepato- and/or splenomegaly. Clinical manifestations of chronic disease result from host immune responses to schistosome eggs. Schistosoma mansoni and S. japonicum eggs most commonly lodge in the blood vessels of the liver or intestine and can cause diarrhea, constipation, and blood in the stool. Chronic inflammation can lead to bowel wall ulceration, hyperplasia, and polyposis and with heavy infections to liver fibrosis and portal hypertension (CDC: Center for Disease Control and Prevention 2018).

An estimated $85 \%$ of the world's cases of schistosomiasis are from Africa, where prevalence rates can exceed $50 \%$ in local populations. Although schistosomiasis had been eliminated in Europe for decades, transmission of S. haematobium was reported

(C) The Author(s). 2019 Open Access This article is distributed under the terms of the Creative Commons Attribution 4.0 International License (http://creativecommons.org/licenses/by/4.0/), which permits unrestricted use, distribution, and reproduction in any medium provided you give appropriate credit to the original author(s) and the source, provide a link to the Creative Commons license, and indicate if changes were made. 
Table 1 Geographical distribution of schistosomiasis species (https://www.who.int/schistosomiasis/ epidemiology/table3/en/)

\begin{tabular}{lll}
$\begin{array}{l}\text { Type of } \\
\text { schistosomiasis }\end{array}$ & Species & Geographical distribution \\
\hline Intestinal schistosomiasis & Schistosoma mansoni & $\begin{array}{l}\text { Africa, the Middle East, the Caribbean, } \\
\text { Brazil, Venezuela, Surinam } \\
\text { China, Indonesia, the Philippines }\end{array}$ \\
& $\begin{array}{l}\text { Schistosoma japonicum } \\
\text { Schistosoma mekongi }\end{array}$ & $\begin{array}{l}\text { Several districts of Cambodia and the } \\
\text { Lao People's Democratic Republic }\end{array}$ \\
& $\begin{array}{l}\text { Schistosoma guineensis and } \\
\text { related S. intercalatum }\end{array}$ & Rain forest area of Central Africa \\
& Schistosoma haematobium & Africa, the Middle East \\
\hline
\end{tabular}

in Corsica in 2014, when cases were identified among travelers who had swimmed in the Cavu River (Montgomery 2017).

\section{Case presentation}

A 36-year-old Eritrean man who had lived in Denmark for the past 3 years presented to the hospital with complaints of 4 months' history of abdominal pain and back pain, intermittent fever, productive cough with hemoptysis (just once), nausea, vomiting, and weight loss of $12 \mathrm{~kg}$. The abdominal pain was mild to moderate, crampy in nature, and generalized. The patient neither had skin rash, chronic diarrhea, or rectal bleeding nor had he traveled/been to Eritrea for the past 3 years. Cardiac and pulmonary examinations were unremarkable while the abdominal exam showed signal of low-grade ascites. There was white blood cell elevation, high levels of CRP, and liver enzymes. Serologies for HIV and hepatitis virus B and C, gonorrhea, and syphilis were negative. Chest X-ray was completely normal without infiltrations or effusions. Abdominal ultrasonography reported fluid accumulation and periportal fibrosis with normal conditions in the liver and spleen and normal portal pressure. Expectorant culture and blood culture were negative for infections. Tuberculosis quantiferon PCR for differential diagnosis was positive, but direct microscopic examination of ascites fluid revealed no tuberculosis bacilli, and acid-fast stain was negative. Computed tomography (CT) of the thorax and abdomen reported normal conditions in the lungs but thickening of the terminal ileum with surrounding inflammatory change. Biopsy from the terminal ileum showed ulcer without necrotic granuloma in intestinal mucosa. Examination of stool and urine for ova was positive for Schistosoma. FDG-PET/CT scan showed enlarged lymph nodes below the diaphragm, widespread foci in peritoneum, omentum majus, and terminal ilium with increased metabolism as well as ascites with up to moderately increased metabolism.

\section{Clinical pathway}

In our study, a diagnostic protocol including routine clinical, biochemical, microbiological, serological, and radiological examinations was performed and ultimately the patient followed up FDG-PET/CT scan in order to determine the etiology of the patient's complex clinical feature. Patient data were retrieved from the hospital records (Table 2). 
Table 2 The clinical pathway of the patient

\begin{tabular}{|c|c|c|}
\hline Date & Tests & Results \\
\hline \multirow[t]{3}{*}{$01 / 05 / 2018$} & White blood cell count & Elevation \\
\hline & Cardiac and pulmonary examinations & Unremarkable \\
\hline & Chest X-ray & Normal \\
\hline 07/05 & CT scan of thorax and abdomen & Thickening of terminal ileum \\
\hline \multicolumn{3}{|c|}{ Early diagnosis: suspect to IBD with cholecystitis } \\
\hline 08/05 & Ultrasonography of the abdomen & Low-grade ascites \\
\hline \multicolumn{3}{|c|}{ Further diagnosis: suspect to Crohn disease } \\
\hline \multirow[t]{3}{*}{ 09/05 } & $\begin{array}{l}\text { Feces examination for } \mathrm{TB}_{1} \\
\text { calprotectin, and pathogens }\end{array}$ & Negative \\
\hline & $\begin{array}{l}\text { Serological tests for HIV, HBV, } \\
\mathrm{HCV} \text {, and syphilis }\end{array}$ & Negative \\
\hline & $\begin{array}{l}\text { Acid-fast staining of expectorant } \\
\text { culture and blood culture }\end{array}$ & Negative \\
\hline \multirow[t]{2}{*}{$12 / 05$} & $\begin{array}{l}\text { Feces examination for intestinal } \\
\text { and skeletal TB }\end{array}$ & Suspect \\
\hline & Tuberculosis quantification PCR for differential diagnosis & Positive \\
\hline \multicolumn{3}{|c|}{ Later diagnosis: suspect to intestinal TB and start of treatment } \\
\hline $16 / 05$ & PET/CT of whole body scan & $\begin{array}{l}\text { Enlarged lymph nodes, widespread foci } \\
\text { in peritoneum, omentum majus, and } \\
\text { terminal ilium and ascites. }\end{array}$ \\
\hline 18/05 & Biopsy of intestinal mucosa and ascite fluid & Ulceration, giant cell, granular \\
\hline 25/05 & Investigation of Schistosomas ova in stool and urine & Positive only in feces \\
\hline 26/05 & Ultrasonography of abdomen for periportal fibrosis & Positive \\
\hline $30 / 05$ & PCR for Schistosomas DNA of stool and urine & Positive only in feces \\
\hline \multicolumn{3}{|c|}{ Final diagnosis: schistosomiasis and start of treatment } \\
\hline
\end{tabular}

Details are described in text

\section{FDG-PET/CT diagnostic procedure}

Injected dose $18 \mathrm{~F}-\mathrm{FDG}$ was $224 \mathrm{MBq}$, uptake time $58 \mathrm{~min}$. The PET scan was performed on a Discovery STE PET-CT scanner from GE (Chicago, IL). The PET scan was decay- and attenuation-corrected using simultaneously and reconstructed using 3D iterative reconstruction with 28 subsets, 2 iterations into $2.5-\mathrm{mm}$ slices. The uptake value (SUV) was standardized using decay-corrected injected dose and total body weight and the low-dose CT $(120 \mathrm{kV})$ was performed (Fig. 1).

\section{Discussion}

Schistosomiasis is usually diagnosed by microscopic identification of parasite eggs in stool (S. mansoni or S. japonicum) or urine (S. haematobium). Since egg shedding may not be consistent such as in travelers and in others who have not had schistosomiasis previously, serologic tests are useful to diagnose light infections (Susan Montgomery 2017). Antibody tests do not distinguish between past and current infections. Immunologic test sensitivity and specificity vary, depending on the antigen preparation used and how the test is performed.

Positron emission tomography (PET) is a functional diagnostic imaging technique using compounds labeled with positron-emitting radioisotopes to measure cell metabolism. [18F] FDG-PET has been used to assess alterations in glucose metabolism in the brain, cancer, cardiovascular diseases, and central nervous system disorders as well as 


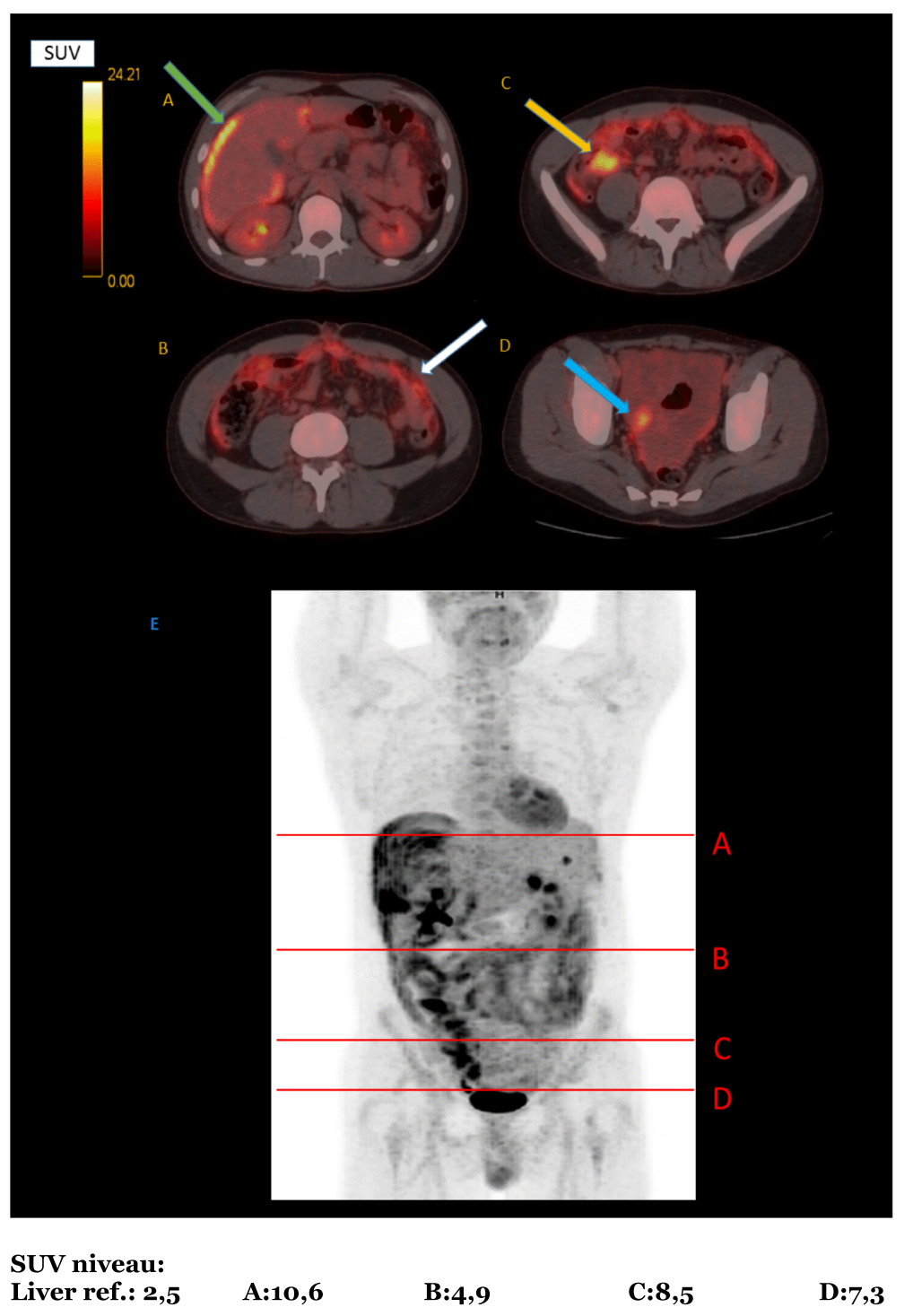

Fig. 1 FDG-PET/CT scans showing foci with pathologic FDG-uptake. (a) Surface of the liver (green arrow). (b) Omental uptake (white arrow). (c) Colonic uptake (yellow arrow). (d) Pelvic ascites (blue arrow). (e) Levels of the images shown in (a)-(d) (FDG standardized uptake value scale shown)

in infectious, autoimmune, and inflammatory diseases (Phelps 2000), and has been evaluated in the diagnostic make-up for fever of unknown origin (Kouijzer et al. 2018), where foci apparent on FDG-PET may not at all be visible on a contrast-enhanced CT.

E. Bueding performed the first worm physiology experiments in 1950 and found schistosomes to be demanding consumers of glucose (Bueding 1950; Salem et al. 2010). There has been only one previous report of using PET/CT to assess Schistosome burden by using in vivo imaging in mice (Salem et al. 2010). They reported that 18F-FDGPET/CT could give clinicians and researchers a quantitative and visual tool to characterize the worm burden in infected individuals, determine the efficacy of a candidate vaccine, and provide information about parasite migration patterns and basic biology. Usually, schistosomal infections are diagnosed mainly by parasitological, serological, or molecular methods, but several imaging modalities have been employed to examine 
schistosomes and monitor schistosome-induced pathology in infected animals including 18F-FDG-PET/CT (Skelly 2013).

Here we are introducing a rare case of intestinal schistosomiasis in a patient with African background with 4 months' history of nonspecific abdominal pain and 12-kg weight loss, and schistosome eggs were found in his stool and urine. Our 18F-FDG-PET/CT scan showed enlarged lymph nodes below the diaphragm, widespread foci in peritoneum, omentum majus, and terminal ilium with increased metabolism and ascites with up to moderately increased metabolism. Intestinal schistosomiasis is very scarcely reported in Europe, but it is important to take into account the differential diagnosis for acute unspecific abdominal pain and weight loss, because of increasing international traveling and disease globalization.

The patient who was diagnosed with schistosomiasis had a complicated clinical history with a severe illness. Standard clinical investigations did not reveal a definite diagnosis but raised a strong suspicion that patient had intestinal tuberculosis with unknown primary source. Eventually, in relation to the patient's country of origin, PET/CT was used at the discretion of physician as part of clinical practice that demonstrated an enlargement of intestinal lymph nodes with increased metabolism widespread foci in peritoneum, omentum majus, and terminal ilium and ascites which was suspected for schistosomiasis infection, but a differential diagnosis could have been peritoneal carcinosis.

It should be noted that some of these tests themselves have limitations in their accuracy because there was no further sampling. In fact, we would have to be able to perform more studies in potentially more populations known to produce relevant results.

\section{Conclusions}

We are suggesting the potential clinical contribution of FDG-PET/CT in the identification of schistosomiasis. We believe that these results are directly applicable to the typical population seen in routine clinical practice. A key question will be how they perform in different population. Studies should be designed in population with a prevalence of schistosomiasis. The classic medical discipline of detailed social and traveling history is crucial for establishing early diagnosis and treatment of exotic infective diseases, but a presumptive diagnosis of schistosome infection may be based on coincidence of FDG uptake in the intestine and lymph nodes below the diaphragm after exclusion of other causes of abdominal pain.

Abbreviations

18F-FDG: F-18-Fluordeoxyglucose; CT: Computed tomography; PET: Position emission tomography

\section{Acknowledgements}

The publication of this article was supported by funds of the European Association of Nuclear Medicine (EANM).

We would like to thank the reviewers who have provided us with helpful comments in this case report.

Authors' contributions

$A D$ and $P M$ were responsible for the data acquisition and analysis. AD, JMG, and PM were responsible for the manuscript drafting and editing. All authors read and approved the final manuscript.

Funding

Not applicable.

Ethics approval and consent to participate

This study was performed in accordance with the ethical standards of the local ethics committee and with the 1964 Helsinki declaration and its later amendments. Images were acquired with clinical indication; patient consent for this retrospective study is waived. 
Received: 8 July 2019 Accepted: 5 September 2019

Published online: 07 October 2019

\section{References}

Bueding E (1950) Carbohydrate metabolism of Schistosoma mansoni. J Gen Physiol 33:475-495

Center for Disease Control and Prevention. Parasites schistosomiasis. (2018). https://www.cdc.gov/parasites/schistosomiasis. Accessed 10 Feb 2019.

Kouijzer LE, Mulders-Manders CM, Bleeker-Rovers CP, Oyen WJG (2018) Fever of unknown origin: the value of FDG-PET/CT. Semin Nucl Med 48(2):100-107 10.1053/j.semnuclmed.2017.11.004

Susan Montgomery. Center for disease control and prevention. Infectious disease related to travel. Chapter3. 2017. Available at: https://wwwnc.cdc.gov/travel/yellowbook/2018/infectious-diseases-related-to-travel/schistosomiasis. Accessed 17 Mar 2019.

Phelps ME (2000) PET: the merging of biology and imaging into molecular imaging. J Nucl Med 41(4):661-681

Salem N, Balkman JD, Wang J et al (2010) In vivo imaging of schistosomes to assess disease burden using positron emission tomography (PET). PLoS Negl Trop Dis 4(9):e827. https://doi.org/10.1371/journal.pntd.0000827.

Skelly PJ (2013) The use of imaging to detect schistosomes and diagnose schistosomiasis. Parasite Immunol 35(9-10):295-301. https:/doi.org/10.1111/pim.12040

\section{Publisher's Note}

Springer Nature remains neutral with regard to jurisdictional claims in published maps and institutional affiliations.

Submit your manuscript to a SpringerOpen ${ }^{\circ}$ journal and benefit from:

- Convenient online submission

$\checkmark$ Rigorous peer review

- Open access: articles freely available online

- High visibility within the field

- Retaining the copyright to your article 